\title{
ALDH1A3 serves as a predictor for castration resistance in prostate cancer patients
}

Shangqian Wang ${ }^{1 \dagger}$, Xiang Zhou ${ }^{1 \dagger}$, Chao Liang ${ }^{1 \dagger}$, Meiling Bao ${ }^{2}$, Ye Tian ${ }^{1}$, Jundong Zhu ${ }^{3}$, Tongtong Zhang ${ }^{1}$, Jie Yang ${ }^{1 *}$ (D) and Zengjun Wang ${ }^{1 *}$

\begin{abstract}
Background: Aldehyde dehydrogenase $1 \mathrm{~A} 3$ (ALDH1A3) has been implicated in the survival and proliferation of prostate cancer cells.

Methods: We retrospectively reviewed our patients with advanced disease on adjuvant hormonal therapy after prostatectomy. Time to castration resistance stage was documented. And Immunohistochemistry analysis for ALDH1A3 was performed for those patient samples on tissue microarray. Bioinformatics anslysis was used for RNA sequencing data of both primary prostate cancer and metastatic castration resistance prostate cancer (mCRPC) from online datasets. Crispr-Cas9 was used to knock out ALDH1A3 in prostate cancer luminal cells, and morphologic analysis as well as the Gene Set Enrichment Analysis (GSEA) were facilitated to discover the mechanisms of the resistance phenotype.

Results: We found that the patients with ALDH1A3 low expression had shorter time to progression to castration resistance compared with those of higher expression group on adjuvant hormonal therapy after radical prostatectomy. The ALDH1A3 knockout cells gradually acquired resistance to androgen deprivation therapy, a few cells have been found in knockout group showing as that the spindle-like luminal cells in charcoal stripped medium. Furthermore, PI3K pathway activation has been confirmed by Western blot. The PI3K pathway inhibitor BEZ235 has been demonstrated that the acquired ADT resistance by ALDH1A3 down regulation could be rescued by PI3K pathway inhibitor.
\end{abstract}

Conclusion: These results suggested a novel function for ALDH1A3 in development of MCRPC, and indicated PI3K pathway inhibitor has the potential in the treatment of a subgroup of mCRPC patients.

Keywords: Castration resistance prostate cancer, Survival time, Aldehyde dehydrogenase, Androgen deprivation therapy, Resistance

\section{Background}

Androgen deprivation therapy (ADT) is the standard of care for advanced prostate cancer or progression after

\footnotetext{
* Correspondence: nanjing_urology@126.com; zengjunwang@njmu.edu.cn †Shangqian Wang, Xiang Zhou and Chao Liang contributed equally to this work

'Department of Urology, The First Affiliated Hospital of Nanjing Medical University, Nanjing, 300 Guangzhou Road, Nanjing 210029, China Full list of author information is available at the end of the article
}

localized definitive treatment. However, most patients eventually progress to a condition known as castrationresistant prostate cancer (CRPC), characterized by lack of response to ADT. Despite the several treatment options for this stage of disease, the impact on overall survival is less than optimal and, most importantly, there is no reliable biomarker to predict the response of the treatment or resistance. As a result, no standard

C C The Author(s). 2020 Open Access This article is licensed under a Creative Commons Attribution 4.0 International License, which permits use, sharing, adaptation, distribution and reproduction in any medium or format, as long as you give appropriate credit to the original author(s) and the source, provide a link to the Creative Commons licence, and indicate if changes were made. The images or other third party material in this article are included in the article's Creative Commons licence, unless indicated otherwise in a credit line to the material. If material is not included in the article's Creative Commons licence and your intended use is not permitted by statutory regulation or exceeds the permitted use, you will need to obtain permission directly from the copyright holder. To view a copy of this licence, visit http://creativecommons.org/licenses/by/4.0/ The Creative Commons Public Domain Dedication waiver (http://creativecommons.org/publicdomain/zero/1.0/) applies to the data made available in this article, unless otherwise stated in a credit line to the data. 
guidance is available to optimally sequence approved treatments for individual patients. Since 2005, the nextgeneration sequencing (NGS) technologies [1] have made it possible for us to better understand the molecular profiles of the cancer, which would provide evidence for clinical practice in oncology, such as diagnosis, prognosis, and treatment decisions. In prostate cancer, the Cancer Genome Atlas (TCGA) has revealed a molecular taxonomy of 333 primary prostate cancer [2]. In addition, the Stand Up to Cancer (SU2C) prostate cancer Dream Team also sequenced 150 metastatic castration resistant prostate cancer samples [3], which benefits a lot to investigate the mechanisms of castration resistance in this disease.

The aldehyde dehydrogenase family 1 member A3 (ALDH1A3) catalyzes the oxidation of retinal to the pleiotropic factor retinoic acid using nicotinamide adenine dinucleotide (NAD+). The level of ALDHs enzymatic activity has been regarded as a cancer stem cell (CSC) marker and seems to correlate with tumor aggressiveness [4] which has been investigated in pancreatic cancer [5], ovarian cancer [6], breast cancer [7], and high-grade glioma [8]. From our previous report [9], we found that ALDH1A3 highly expressed in the human prostate, specially in the luminal compartment. In the primary prostate cancer, the expression of this gene correlated with AR pathway and luminal markers. Furthermore, for those with advanced disease after radical prostatectomy who underwent adjuvant androgen deprivation therapy, negative ALDH1A3 expression predicted as shorter time for castration resistance upon hormonal therapy.

We found, surprisingly, that ALDH1A3 was down regulated in metastatic castration resistant prostate cancer from previous sequencing data [10]. Combing with our previous report, the low expression of ALDH1A3 might be related with regression of AR signaling pathway at castration condition. Strengthening with the proof reanalyzed from RNA sequencing of $150 \mathrm{mCRPC}$ patients, ALDH1A3-low group seems to be related with lymph nodes metastases, and activation of PI3K pathway signaling. Furthermore, we confirmed this hypothesis with experiments, supporting that ALDH1A3 null could facilitate prostate cancer cells in castrated condition via PI3K pathway, but could be rescued by PI3K pathway inhibitor. These results provide evidence that ALDH1A3 could be a potential biomarker of castration resistant prostate cancer, supporting future clinical trial on overcoming the ADT resistance.

\section{Methods}

\section{Patients and tissue microarrays}

The protocol to generate the tissue microarrays (TMAs) in this cohort has been described in our previous report [9]. We retrospectively reviewed our patients with advanced disease on adjuvant hormonal therapy after prostatectomy. A total of 79 patients in our single center were included in this study. Those who has lost followup or benign tissue on the TMA were excluded. All these patients were performed laparoscopic radical prostatectomy with positive lymph nodes or positive margins followed by adjuvant hormonal therapy (LHRH analogs and bicalutamide as standard of care) between 2012 and 2014 at the urology department of the First Affiliated Hospital of Nanjing Medical University. All patients were recruited following informed consent, the protocol was approved by ethical committee of The First Affiliated Hospital of Nanjing Medical University. Progression to castration resistant prostate cancer (CRPC) defined as biochemical recurrence or metastasis on adjuvant hormonal therapy. For the staining score system, we have described the protocol in our previous report [9]. Briefly, For the staining score system [11], the percentage of positive tumor cells was determined by at least five areas at 400 magnification and assigned to one of the following five categories: $0<5 \% ; 1: 5-25 \%$; $2: 25-50 \%$; $3: 50$ $75 \%$, and $4:>75 \%$. The intensity of immunostaining was scored as follows: 1 low, 2, moderate, and 3, strong. The IHC score for ALDH1A3 on prostate cancer slides was: low expression $<8$, and high expression $\geq 8$.

\section{Database and bioinformatics}

Three datasets (Cornell [12], MSKCC [13], Michigan group [10]) on prostate cancer samples sequencing profiles were found and the RNA sequencing data in RPKM format were downloaded. The ALDH1A3 expression value for each samples in mCRPC group and primary cancer group were compared in each dataset. The results were shown by GraphPad software.

\section{Gene set enrichment analysis (GSEA) analysis}

The RNA sequencing data were downloaded from SU2C database. The median value of RPKM for ALDH1A3 was used as cut-off value, any sample which is higher than the median value was determined as ALDH1A3 $3^{\text {high }}$, the lower samples as ALDH1A3 ${ }^{\text {low }}$. The GSEA analysis was performed according to the protocol which was previously described [14]. The Genesets were downloaded from the Molecular Signatures Database (MSigDB http://software.broadinstitute.org/gsea/msigdb/).

\section{Cell culture and Crispr-Cas9 knockout}

The human prostate cancer cell line ( $\mathrm{LnCaP}, \mathrm{VCaP}$ ) were purchased from the Cell Bank Type Culture Collection of the Chinese Academy of Sciences (Shanghai, China) and maintained in RPMI medium with $10 \%$ fetal bovine serum within a humidified atmosphere containing $5 \% \mathrm{CO} 2$ at $37^{\circ} \mathrm{C}$. 
We designed the guide RNA for ALDH1A3 from (http://crispr.mit.edu/), targeting the first exon. The sequence of the guide is as follows-ALDH1A3: 1- AGTTATGGCTACCACCAACG; 2-TAGTCTGCGGCGCA CCGGCT; green fluorescent protein (GFP): GGCGAG GAGCTGTTCACCG. Then, we ligated the guide to the LentiCrispr-V2 system followed by Sanger sequencing validation [15]. Finally, we produced the lentivirus according to the protocol previously described [9]. After 2 days of the infection to the $\mathrm{LnCaP}$ and $\mathrm{VCaP}$ cells, the puromycin selection was performed. We performed Western Blot assay to validate the knockout efficiency after 14 days of infection. The cell numbers were counted based on the typan blue staining.

\section{Western blotting}

The protein expression of ALDH1A3 by Western Blot assay was performed according to the protocol previously described. The antibodies against ALDH1A3 (Abcam, USA), Phospho-Akt (Ser473, Cell Signaling Technology, USA) and glyceraldehyde 3-phosphate dehydrogenase (GAPDH; Bioworld Technology, Inc., USA) were used in Western Blot assay in accordance with the manufacturer's instructions.

\section{Statistical analysis}

Differences in vitro experiment like cell numbers between groups were subjected to Student's test. $p<0.05$ was considered to be statistically significant. All the statistical calculations were performed using GraphPad Prism v6.0 software (GraphPad Prism version 6.00 for Windows, GraphPad Software, La Jolla California USA, www.graphpad.com).

\section{Results}

ALDH1A3 negative predicted CRPC in patients on adjuvant ADT

Our earlier work has demonstrated that ALDH1A3 highly expressed in human prostate, which had a strong correlation with primary prostate cancer luminal signature and could be a potential biomarker of AR signaling pathway. Then we moved on to investigate its expression in the castration resistant prostate cancer. We retrospectively reviewed 79 patients with advanced disease who underwent radical prostatectomy followed by adjuvant hormonal therapy in our center. It was indicated

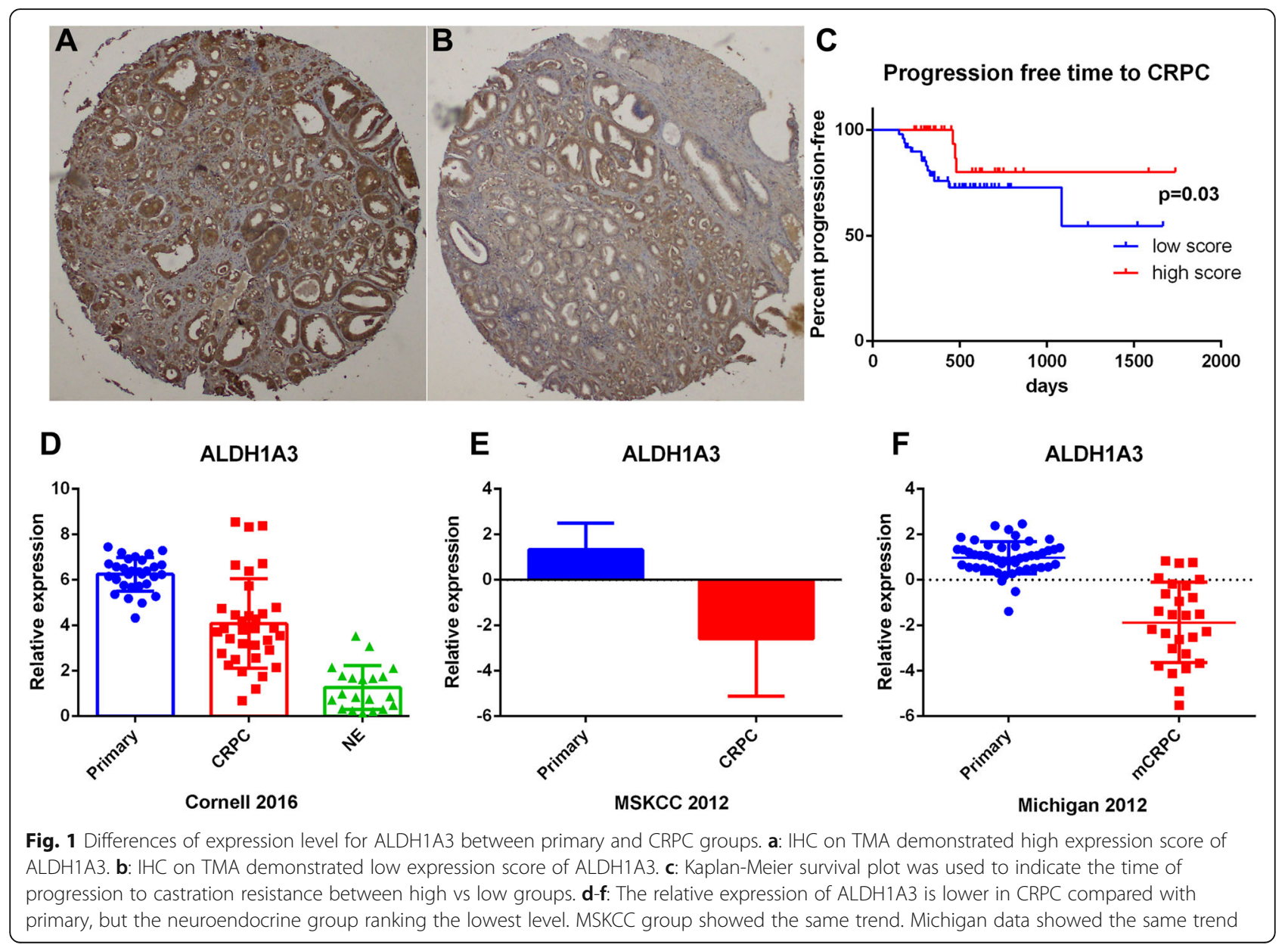


that negative expression of ALDH1A3 predicted shorter time progression to castration resistance on adjuvant hormonal therapy (Fig. 1a-c). Besides, its expression is down regulated in three datasets. Beltran et al. performed a sequencing profiling on 114 metastatic samples from 51 CRPC and 30 neuroendocrine prostate cancer patients. The ALDH1A3 expression was lower in neuroendocrine and CRPC group compared with primary cancer (Fig. 1d). Another group performed RNA sequencing for primary prostate cancer and mCRPC samples, ALDH1A3 also down regulated in mCRPC samples (Fig. 1e). The data from Michigan group showed the same trend (Fig. 1f). All expression level of ALDH1A3 in these three datasets are defined as $\mathrm{Z}$ score or $\log 2$ value.

\section{ALDH1A3 signature has a strong correlation with prostate} cancer progression, and PI3K signaling pathway

A multi-institutional clinical sequencing infrastructure to conduct prospective transcriptome sequencing of bone or soft tissue tumor biopsies from a cohort of 150
mCRPC affected individuals was performed to establish a precision medicine framework for $\mathrm{mCRPC}$. According to the expression level of ALDH1A3 in the dataset, we defined the cases above the median value of ALDH1A3 in the whole cases as ALDH1A3 ${ }^{\text {high }}$, those whose expression level lower than the median as ALDH1A3 $3^{\text {low. We }}$ compared ALDH1A3 $3^{\text {high }}$ and ALDH1A3 ${ }^{\text {low }}$ cases to generate a differential expression gene list as ALDH1A3 signature (Table S1). The ALDH1A3 ranking the most significant changes in the whole list confirmed the results. The Gene Set Enrichment Analysis (GSEA) finally correlated ALDH1A3 signature with several biologic event. We found the ALDH1A3 signature had significant positive correlation with ERG signature and prostate cancer luminal signature, respectively (Enrichment score: 0.77 , 0.5; Both $p$ value: <0.01) (Fig. 2a, b), and it had negative correlation with lymph nodes and PI3K-AKTmTOR signaling pathway, meaning that ALDH1A3 ${ }^{\text {low }}$ group might be associated with lymph nodes metastasis and PI3K-AKT-mTOR signaling activation (Fig. 2c, d).

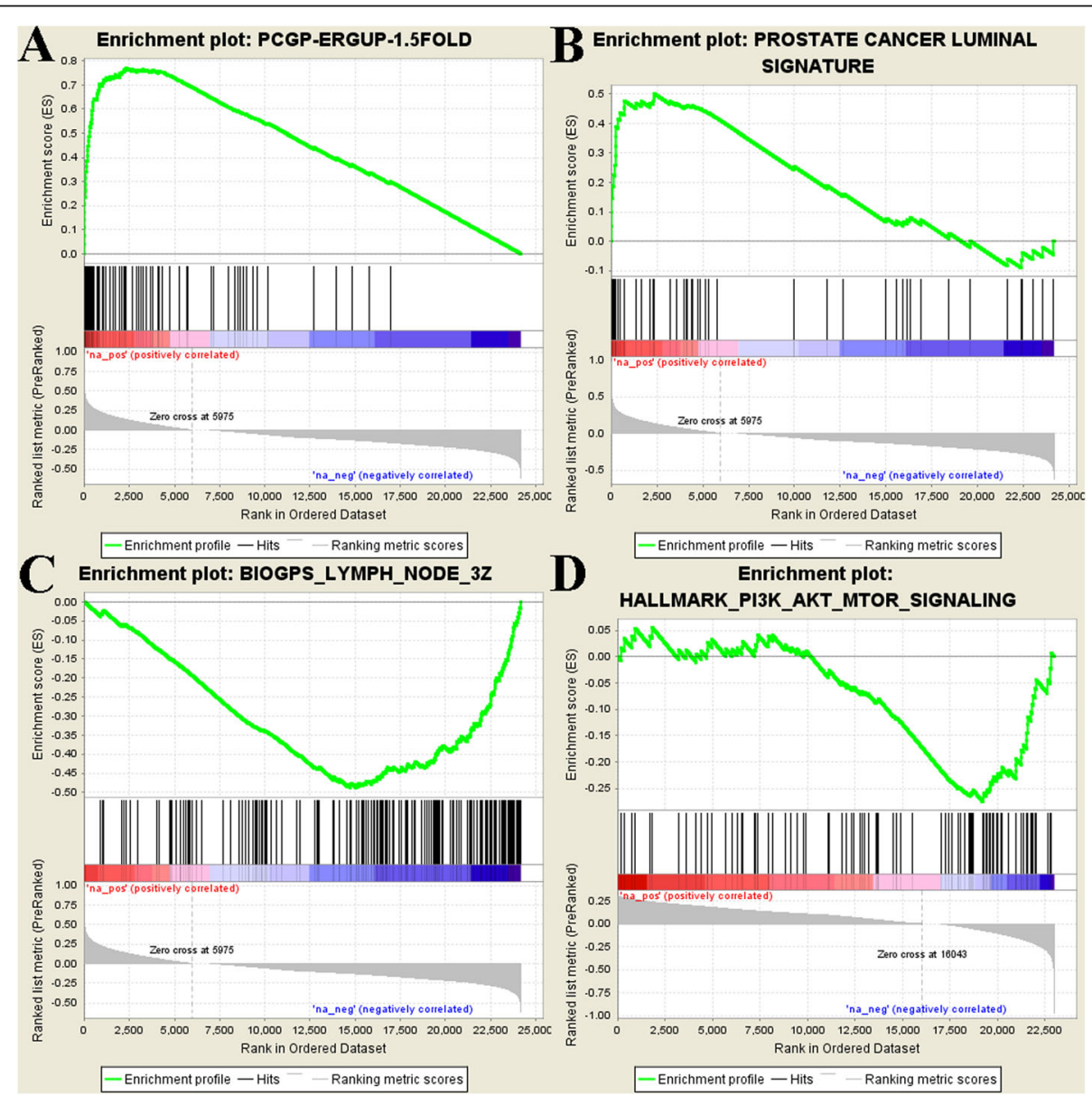

Fig. 2 GSEA analysis for ALDH1A3 signature. a: ALDH1A3 has positive correlation with ERG up regulation. b: ALDH1A3 has positive correlation with prostate cancer luminal signature. $\mathbf{c}$ : ALDH1A3 has negative correlation with lymph node. $\mathbf{d}$ : ALDH1A3 has negative correlation with PI3K-AKT-mTOR signaling 


\section{Down-regulation of ALDH1A3 causes ADT resistance in prostate cancer cells}

Based on the above results, we aimed to investigate the mechanisms of negative expression of ALDH1A3 in mCRPC samples. We designed small guide RNA targeting the functional exon of ALDH1A3 to knock out this gene on cell level to see the phenotype changes. After validation of the knock out efficiency (Fig. 3a, Fig. S1-2), we picked up the most potent sgRNA to target ALDH1A3 in LnCaP and VCaP cells, both of which are sensitive to androgen ablation treatment in vitro. We found that the growth rate of the control cells (targeting GFP) had no significant difference with ALDH1A3 knockout cells in normal medium. But in charcoal stripped medium which has already filtered androgen, the ALDH1A3 knockout cells, growing slowly, could be able to survive. As a result, the growth rate of ALDH1A3 knockout cells was significantly faster than the control cells in charcoal stripped medium (Fig. 3b). We also repeated the experiment in $\mathrm{VCaP}$ cells, and the results were consistent with those in LnCaP cells (Fig. 3b). We did the morphology observation for those LnCaP cells as well to predict the potential mechanisms of the ADT resistance. From Fig. 3c, At day 7, the control cells in medium without DHT showed spindle-like morphology and the cell number is low compared with those control cells in normal medium showing in aggregation or in cluster. However, it didn't show any difference in ALDH1A3 knockout cells in DHT-free medium and in normal medium. At day 14, 95\% of the control cells in DHT-free medium had been dead, whereas there were $30-40 \%$ of the ALDH1A3 knockout cells still alive.

\section{ALDH1A3 knockout facilitates castration resistance through PI3K-AKT-mTOR signaling pathway}

Based on the above GSEA results, the ALDH1A3 signature negatively correlated with PI3K-AKT-mTOR signaling pathway. We speculate that the ALDH1A3 loss could activate the PI3K pathway. In order to validate this hypothesis, we did Western blot assay to test the PI3K pathway activation to compare the ALDH1A3 wild type cells and knockout cells. The blotting showed that both in $\mathrm{LnCaP}$ and $\mathrm{VCaP}$ cells, phospho-AKT had been elevated in ALDH1A3 knockout group (Fig. 4, Fig. S3, 4, 5). Next, in order to determine the relationship between castration resistance and PI3K signaling pathway activation by ALDH1A3 knockout, We performed rescue assay to block PI3K signaling pathway by using PI3K signaling inhibitor BEZ235. The results demonstrated that $500 \mathrm{nM}$ BEZ235 treatment after $48 \mathrm{~h}$ could rescue the resistance by ALDH1A3 knockout. In terms of the morphology analysis, LnCaP cells in BEZ235 treatment and DHT-free group showed spindle-like morphology, similar with control cells in the DHT-free medium (Fig. 5a). Those cells showed less aggressive phenotype and finally detached from the bottom of the plate. The growth rate of ALDH1A3 knockout cells showed 30\%

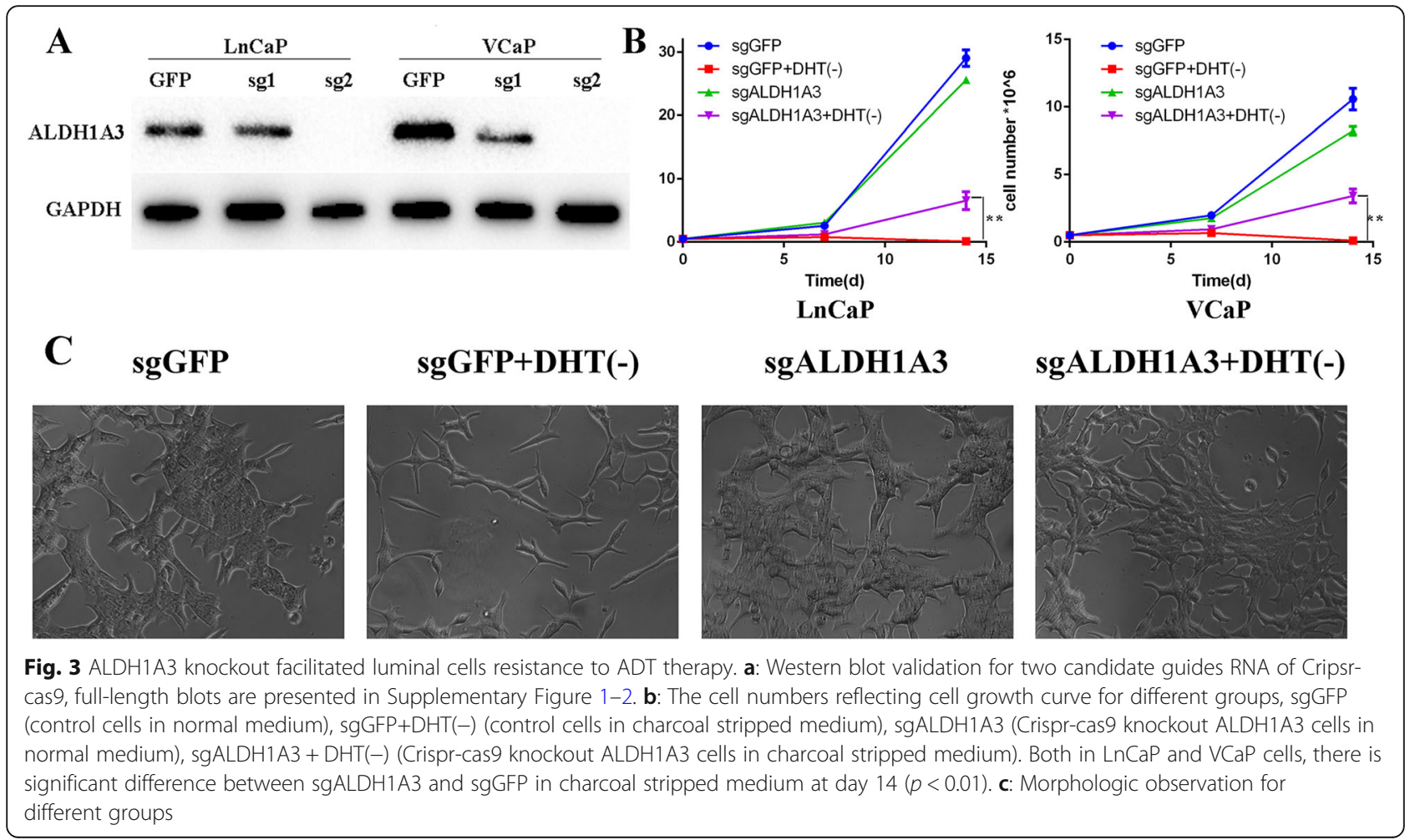




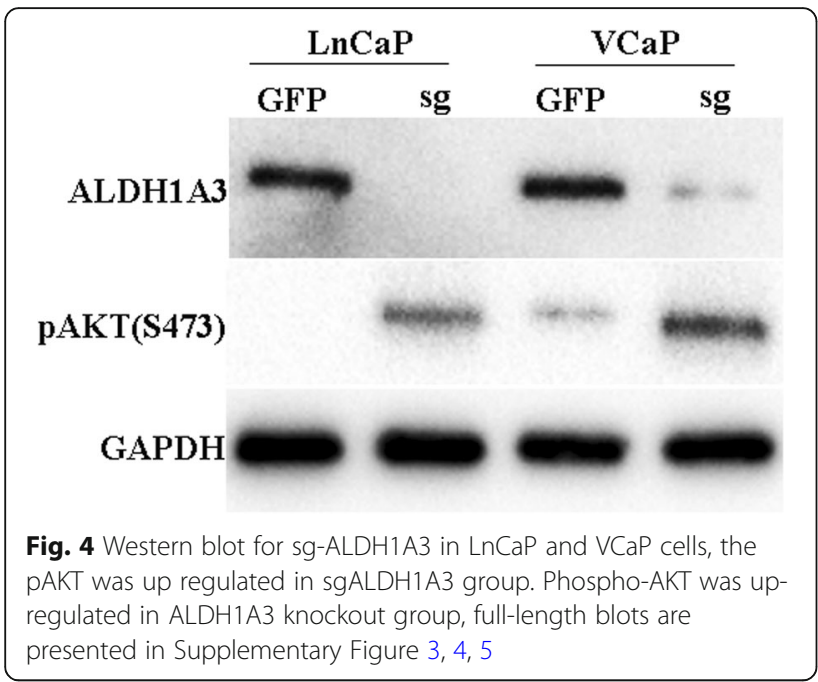

higher than the wild type cells but was inhibited totally by BEZ235 in DHT-free medium (Fig. 5b).

\section{Discussion}

Our previous study has demonstrated that ALDH1A3 is specifically expressed in luminal compartment in human prostate epitheliums. From the TCGA data of 333 primary prostate cancer, ALDH1A3 correlated with AR signaling pathway and corresponding luminal signature. It is also suggested that ALDH1A3 has a potential to be a predictor of survival in primary prostate cancer patients. In present study, with our single center follow up database, we performed IHC analysis on tissue microarray for patients with advanced disease upon adjuvant hormonal therapy after radical prostatectomy. The results showed that ALDH1A3 low-expression patients indicated shorter time to progression to castration resistance. The phenotype that we showed at the beginning has implications to understand the mechanisms of this prostate specific gene in the development of cancer progression. From the metastatic prostate cancer samples database, the RNA sequencing data demonstrated that ALDH1A3 was down regulated in MCRPC group compared with the primary prostate cancer. Nextgeneration sequencing (NGS) analysis has made it possible to reclassify different subtypes in a specific cancer by molecular changes. It indeed could provide benefits for clinical practice in oncology, such as diagnosis, prognosis, and treatment decisions. For example, patients with cancers of unknown primary (CUP), traditionally, are generally assumed to have a poor prognosis with a treatment in cytotoxic chemotherapy guided by histologic features and the pattern of metastatic spread. A new study showed that NGS may provide an opportunity for CUP patients to benefit from individualized therapies according to the targetable genomic alterations identified by tumor
A sgGFP+DHT(-)
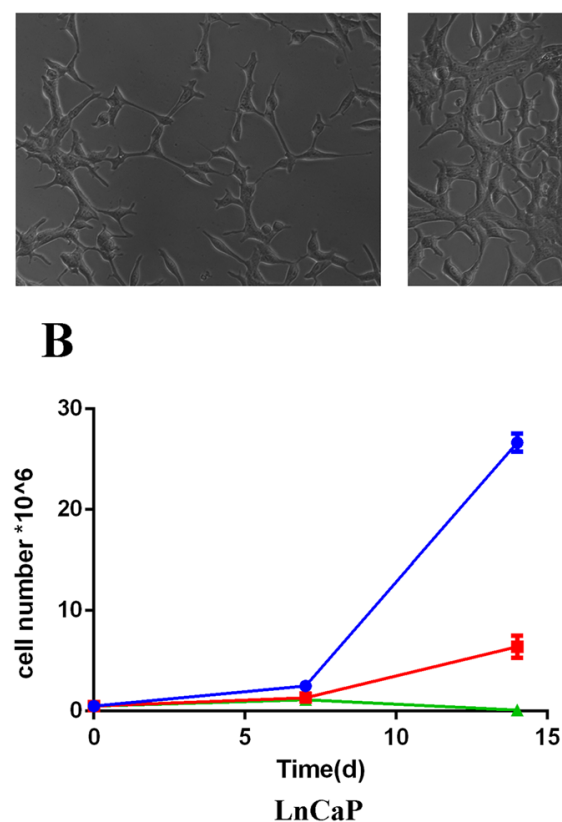

$\operatorname{sgALD+DHT(-)} \quad \operatorname{sgALD+DHT(-)+BEZ235}$
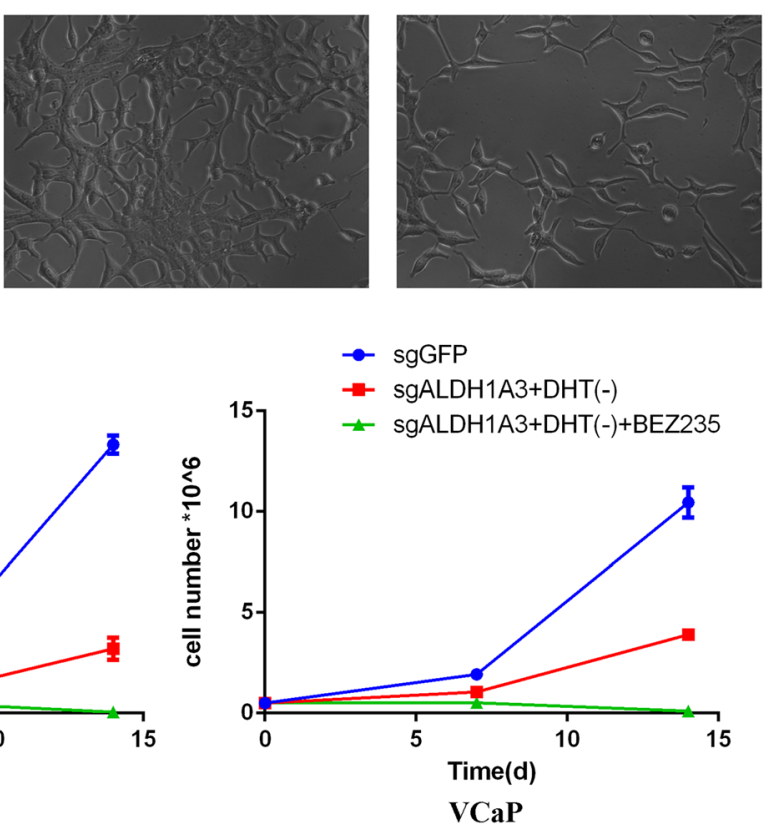

Fig. 5 BEZ235 treatment rescued the resistance through PI3K pathway inhibition. a: Morphologic observation for BEZ235 treatment compared with the resistance phenotype. $\mathbf{b}$ : The growth curve documented the cell numbers of different groups, BEZ235 treatment rescued the ADT resistance in sg-ALDH1A3 group 
molecular profiling [16]. In that study, $10 \%$ of patients received targeted therapies based on their mutation signatures.

Thanks to the RNA sequencing data, we found that the PI3K pathway signature had been highly correlated with ALDH1A3 signature. Then we speculated the PI3K signaling pathway activation might be due to ADT resistance. And we also confirmed this hypothesis by showing the up-regulation of phospho-AKT upon ALDH1A3 knockout. Furthermore, PI3K signaling pathway inhibitor BEZ235 could rescue the ADT resistance following ALDH1A3 knockout. The PI3K/AKT/mTOR pathway is altered in almost $50 \%$ of mCRPC through either PTEN inactivation or/and aberrant activation in PIK3CA/B [13]. It's been demonstrated that PI3K-AKTmTOR signaling pathway deregulation resulting from PTEN loss is associated with androgen insensitivity and the development of CRPC [17]. Knocking down PTEN can convert the androgen-dependent $\mathrm{Myc}-\mathrm{CaP}$ cell into androgen independence, suggesting that PTEN intrinsically controls androgen responsiveness, a critical step in the development of castration resistant prostate cancer [18]. Based on these data, phase I/II trials assessing the combination of next-generation AR therapy with a $\mathrm{PI} 3 \mathrm{~K} / \mathrm{AKT} / \mathrm{mTOR}$ inhibitor are currently ongoing (ClinicalTrials.gov identifier: NCT02407054 and NCT02215096). ALDH1A3, also as retinoic acid anabolizing enzyme [19] has been proved a potential novel target for triple-negative breast tumors and cancer stem cells [20]. Retinoic acid receptor-related orphan receptor $\gamma(\mathrm{ROR} \gamma)$ antagonists are efficacious in re-sensitizing docetaxel and cabazitaxel cross-resistant CRPC cells [21]. It demonstrated that targeting retinoid signaling might be a potential approach in the treatment of CRPC [22].

\section{Conclusions}

In conclusion, the NGS provides multiple new opportunities and tools to accelerate and facilitate the entire process of drug testing toward accelerated drug positioning and approval for precise and personalized medicine. It also allows researchers to discover some hidden pattern of the complex cancer. Such strategies include the development of inhibitors with a higher potency against their intended target, like ADT and Abiraterone, and the use of combination therapies incorporating inhibitors of parallel or alternative signaling pathways mediating acquired resistance, like the mTOR inhibitor BEZ235 in the treatment of PI3K-AKT-mTOR pathway activation. In this paper, we've investigated alterations in the targeted gene leading to ADT resistance to $\mathrm{mCRPC}$. Based on the RNA sequencing and experimental results, we found that PI3K pathway alteration or activation might be the cause of the resistance. We, then, rescued the ADT resistance by PI3K pathway inhibitor-BEZ235. The acquired resistance to ADT therapy by some patients with low level of ALDH1A3 could be overcome by combination therapy with PI3K pathway inhibitor, which will provide a new potential approach to the treatment of mCRPC.

\section{Supplementary information}

Supplementary information accompanies this paper at https://doi.org/10. 1186/s12885-020-06899-x.

Additional file 1 : Table S1: A differential expression gene list as ALDH1A3 signature.

Additional file $\mathbf{2}$ : Figure S1: Original data of western blot (ALDH1A3) in Fig. 3a, the cropping of the blot by figure processing software was clearly mentioned with red rectangle.

Additional file $\mathbf{3}$ : Figure S2: Original data of western blot (GAPDH) in Fig. 3a, the cropping of the blot by figure processing software was clearly mentioned with red rectangle.

Additional file 4 : Figure S3: Original data of western blot (ALDH1A3) in Fig. 4, the cropping of the blot by figure processing software was clearly mentioned with red rectangle.

Additional file 5 : Figure S4: Original data of western blot ( $p-A K T)$ in Fig. 4, the cropping of the blot by figure processing software was clearly mentioned with red rectangle.

Additional file 6 : Figure S5: Original data of western blot (GAPDH) in Fig. 4 , the cropping of the blot by figure processing software was clearly mentioned with red rectangle.

\section{Abbreviations}

mCRPC: metastatic Castration Resistance Prostate Cancer; GSEA: Gene set enrichment analysis; ADT: Androgen deprivation therapy; TCGA: The cancer genome atlas; SU2C: Stand up to cancer; NAD: Nicotinamide Adenine Dinucleotide; CSC: Cancer stem cell; TMAs: Tissue microarrays; LHRH: Luteinizing hormone-releasing hormone; RPKM: Reads Per Kilobase Million; DHT: Dihydrotestosterone; IHC: Immunohistochemistry; NGS: Nextgeneration sequencing; CUP: Cancers of unknown primary; RORy: Retinoic acid receptor-related orphan receptor $\gamma$

\section{Acknowledgements}

Not applicable.

\section{Authors' contributions}

YJ and WZ designed this work. WS, ZX and LC performed the experiment and wrote the manuscript. BM and TY performed the pathology experiment and data revie. ZJ and ZT performed the follow-up of the patients. All authors have read and approved the manuscript.

\section{Funding}

This study was funded by Prostate cancer cohort study of Nanjing Medical University (NMUC2018003A). Specifically, tissue array, and biochemistry assays were supported by this funding.

Availability of data and materials

The datasets analyzed in this study was included in supplementary Table 1.

Ethics approval and consent to participate

All the patients provided written informed consent, the protocol was approved by ethical committee of The First Affiliated Hospital of Nanjing Medical University.

Consent for publication

Not applicable.

Competing interests

The authors declare no conflict of interests. 


\section{Author details}

'Department of Urology, The First Affiliated Hospital of Nanjing Medical University, Nanjing, 300 Guangzhou Road, Nanjing 210029, China. ${ }^{2}$ Department of Pathology, The First Affiliated Hospital of Nanjing Medical University, Nanjing, China. ${ }^{3}$ Department of Urology, The Third Affiliated Hospital of Soochow University, The First People's Hospital of Changzhou, Changzhou, China.

Received: 15 January 2020 Accepted: 23 April 2020

Published online: 06 May 2020

\section{References}

1. Margulies M, Egholm M, Altman WE, et al. Genome sequencing in microfabricated high-density picolitre reactors. Nature. 2005;437:376-80.

2. Cancer Genome Atlas Research N. The Molecular Taxonomy of Primary Prostate Cancer. Cell. 2015;163:1011-25.

3. Robinson D, Van Allen EM, Wu YM, et al. Integrative clinical genomics of advanced prostate cancer. Cell. 2015;161:1215-28.

4. Moretti A, Li J, Donini S, Sobol RW, Rizzi M, Garavaglia S. Crystal structure of human aldehyde dehydrogenase $1 \mathrm{~A} 3$ complexed with NAD+ and retinoic acid. Sci Rep. 2016;6:35710.

5. Kong B, Wu W, Cheng T, et al. A subset of metastatic pancreatic ducta adenocarcinomas depends quantitatively on oncogenic Kras/Mek/Erkinduced hyperactive mTOR signalling. Gut. 2016;65:647-57.

6. Saw YT, Yang J, Ng SK, et al. Characterization of aldehyde dehydrogenase isozymes in ovarian cancer tissues and sphere cultures. BMC Cancer. 2012;12:329.

7. Ali HR, Dawson SJ, Blows FM, Provenzano E, Pharoah PD, Caldas C. Cancer stem cell markers in breast cancer: pathological, clinical and prognostic significance. Breast Cancer Res. 2011;13:R118.

8. Mao P, Joshi K, Li J, et al. Mesenchymal glioma stem cells are maintained by activated glycolytic metabolism involving aldehyde dehydrogenase 1A3. Proc Natl Acad Sci U S A. 2013;110:8644-9.

9. Wang $\mathrm{S}$, Liang $\mathrm{C}$, Bao M, et al. ALDH1A3 correlates with luminal phenotype in prostate cancer. Tumour Biol. 2017;39:1010428317703652.

10. Grasso CS, Wu YM, Robinson DR, et al. The mutational landscape of lethal castration-resistant prostate cancer. Nature. 2012;487:239-43.

11. Tong X, Li K, Luo Z, et al. Decreased TIP30 expression promotes tumor metastasis in lung cancer. Am J Pathol. 2009;174:1931-9.

12. Beltran $H$, Prandi $D$, Mosquera JM, et al. Divergent clonal evolution of castration-resistant neuroendocrine prostate cancer. Nat Med. 2016;22:298-305.

13. Taylor BS, Schultz N, Hieronymus H, et al. Integrative genomic profiling of human prostate cancer. Cancer Cell. 2010;18:11-22.

14. Gao D, Vela I, Sboner A, et al. Organoid cultures derived from patients with advanced prostate cancer. Cell. 2014;159:176-87.

15. Sanjana NE, Shalem O, Zhang F. Improved vectors and genome-wide libraries for CRISPR screening. Nat Methods. 2014;11:783-4.

16. Varghese AM, Arora A, Capanu M, et al. Clinical and molecular characterization of patients with cancers of unknown primary in the modern era. Ann Oncol. 2017;28(12):3015-21.

17. Wang $\mathrm{S}$, Gao J, Lei $\mathrm{Q}$, et al. Prostate-specific deletion of the murine Pten tumor suppressor gene leads to metastatic prostate cancer. Cancer Cell. 2003:4:209-21.

18. Jiao J, Wang S, Qiao R, et al. Murine cell lines derived from Pten null prostate cancer show the critical role of PTEN in hormone refractory prostate cancer development. Cancer Res. 2007;67:6083-91.

19. Lochbaum R, Schilpp C, Nonnenmacher L, Frick M, Dietl P, Wittekindt OH. Retinoic acid signalling adjusts tight junction permeability in response to air-liquid interface conditions. Cell Signal. 2020;65:109421.

20. Vidovic D, Huynh TT, Konda P, et al. ALDH1A3-regulated long non-coding RNA NRAD1 is a potential novel target for triple-negative breast tumors and cancer stem cells. Cell Death Differ. 2020;27:363-78.

21. Wang $Y$, Huang $Z$, Chen $C Z$, et al. Therapeutic targeting of MDR1 expression by RORgamma antagonists Resensitizes cross-resistant CRPC to Taxane via coordinated induction of cell death programs. Mol Cancer Ther. 2020;19:364-74.

22. Seed RI, Taurozzi AJ, Wilcock DJ, et al. The putative tumour suppressor protein Latexin is secreted by prostate luminal cells and is downregulated in malignancy. Sci Rep. 2019;9:5120.

\section{Publisher's Note}

Springer Nature remains neutral with regard to jurisdictional claims in published maps and institutional affiliations.

\section{Ready to submit your research? Choose BMC and benefit from:}

- fast, convenient online submission

- thorough peer review by experienced researchers in your field

- rapid publication on acceptance

- support for research data, including large and complex data types

- gold Open Access which fosters wider collaboration and increased citations

- maximum visibility for your research: over $100 \mathrm{M}$ website views per year

At BMC, research is always in progress.

Learn more biomedcentral.com/submissions 